\title{
MAPEAMENTO DE PROCESSO E PENSAMENTO ENXUTO: UM ESTUDO BIBLIOMÉTRICO
}

\author{
PROCESS MAPPING AND LEAN THINKING: A BIBLIOMETRIC STUDY \\ Matheus de Jesus Amorim ${ }^{1}$, \& Thiago Padovani Xavier ${ }^{2 *}$ \\ ${ }^{12}$ Centro Universitário Norte do Espírito Santo da Universidade Federal do Espírito Santo. \\ ${ }^{1}$ matheus_amorim741@ hotmail.com ${ }^{2 *}$ thiago.p.xavier@ufes.br
}

\section{ARTIGO INFO.}

\section{Recebido em: 05.03.2021}

Aprovado em: 29.03.2021

Disponibilizado em: 26.05.2021

\section{Palavras-chave:}

VOSviewer; Base de dados; Web of Science.

\section{KEYWORDS:}

VOSviewer; Data base; Web of Science.

\section{*Autor Correspondente: Xavier, T. P.}

\section{RESUMO}

Este artigo analisa as características das publicações relacionadas aos temas mapeamento de processo e pensamento enxuto. A pesquisa foi realizada na base de dados Web of Science, procurando examinar sete áreas temáticas, intituladas: autores; áreas de pesquisa; anos de publicação; organizações; países/regiões; agências financiadoras; e fontes de publicações. Além disso, foi realizado um comparativo entre autores com os maiores números de publicações com os artigos mais citados no período analisado, para verificar a relação entre eles. Durante o período de 2010 até 2019, foram tratados 198 artigos ao todo e, de acordo com os resultados deste estudo, o número de publicações cresceu de forma significativa em boa parte do período estipulado, porém publicações relacionadas à temática do pensamento enxuto apresentaram um declínio em 2019. Ademais, são apresentadas todas as agências financiadoras de pesquisas relacionadas aos temas, sendo de maior destaque as norteamericanas, o que justifica a liderança dos Estados Unidos das Américas no ranking dos países que mais publicam sobre process mapping e lean thinking. Constatou-se, ainda, que a maioria dos autores que se destacaram em quantidade de publicações, não possuem seus trabalhos entre os mais citados, isso é, os artigos de maior impacto nas temáticas pesquisadas.

\begin{abstract}
This article analyzes the characteristics of publications related to process mapping and lean thinking. The research was carried out in the Web of Science database, seeking to examine seven thematic areas, entitled: authors, research areas, years of publication, organizations, countries / regions, funding agencies and sources of publications, in addition, a comparison was made among the most cited publications with the authors who published the most about the researched topics. During the period from 2010 to 2019, 198 articles were treated in all and, according to the results of this study, the number of publications grew significantly in much of the stipulated period, but publications related to the theme of lean thinking showed a decline in 2019. In other words, $86.36 \%$ of the publications were written in the English language, and having as main source the journals related to health, which demonstrates the scope of application of the themes. It was also found that most authors who stood out in the number of publications do not have their work among the most cited, that is, the articles with the greatest impact on the themes studied.
\end{abstract}




\section{INTRODUÇão}

No cenário atual, em que o mercado se encontra cada vez mais globalizado, as empresas almejam se tornar referência no setor em que atuam. Um dos principais meios para se sobressair é com desenvolvimento de produtos que visam satisfazer as necessidades dos clientes. Nesse contexto, o mapeamento de processo é uma importante ferramenta, pois ela é utilizada para entender de forma clara como uma unidade de produção está funcionando, demonstrando cada passo de operação dessa unidade por meio de entradas, saídas e ações (Aguiar, Damasceno \& Melo, 2016). Com isso, após a leitura e análise do funcionamento da linha de produção é possivel, estabelecer medidas para melhorar a produtividade, qualidade e eficiência da empresa, para tal, pode-se utilizar o pensamento enxuto que é uma filosofia de trabalho que permite diminuir os desperdícios durante a elaboração e execução de um produto, melhorando os resultados no atendimento às necessidades dos clientes (Aherne \& Whelton, 2010).

O mapeamento de processo auxilia a empresa a identificar seus pontos fortes, fracos e que podem ser melhorados tais como: reduzir custos; gargalos; falhas de integração; retrabalhos; entre outros. Desta forma, é possível melhorar o entendimento sobre os processos e aumentar a performance do negócio. Ainda, o mapeamento de processo pode ser compreendido como modelagem de processos, por meio de representações gráficas que possibilitam a análise desses, utilizando as informações a respeito das atividades, setores e profissionais envolvidos na execução dos processos, bem como a integração entre todos os processos realizados (Hörbe Moura, Silva, Vargas \& Machado, 2015; Gonçalves, Castro, Cruvinel, Jesus, Siqueira \& Sousa, 2021).

Por meio dos estudos da Massachusetts Institute of Technology (MIT) sobre a indústria automobilística mundial, no período de 1985 a 1990, surgiu o conceito de enxuto. Esse conceito foi utilizado para explicar o Sistema Toyota de Produção (STP), um sistema de produção de automóveis desenvolvido após a Segunda Guerra Mundial. Tal sistema difundiu uma nova prática para os processos produtivos, com o objetivo de melhorar os níveis de produção e eficiência através da eliminação de desperdício e a utilização de melhorias contínuas (Womack, Jones \& Roos, 2004; Karim \& Uz-Zaman, 2013).

Buscando analisar a relevância destes temas no meio cientifico, foi utilizado a bibliometria que tem por finalidade analisar estudos científicos mediante análise quantitativa das publicações, podendo determinar a relevância que determinado assunto teve em determinado período. Além disso, o estudo bibliométrico consiste na utilização dos métodos estatísticos sobre o conjunto de referências bibliográficas, a fim de mensurar índices de produção acadêmica (Vanti, 2002; Araújo, 2006).

Este estudo, tem por objetivo específico, captar informações acerca das sete áreas temáticas que trazem dados quantitativos sobre o andamento de pesquisas sobre mapeamento de processo e pensamento enxuto, sendo estas: autores; áreas de pesquisa; anos de publicação; organizações; países/regiões; agências financiadoras; e fontes de publicações. Ainda, a justificativa para o referido estudo, se dá pela importância de acompanhar a evolução e analisar a produção científica dos temas abordados. 

Brazilian Journal of Production Engineering, 6(9), Edição Especial "Iniciação Científica", 47-65.

Com essa breve contextualização, o presente estudo objetivou realizar uma análise bibliométrica associada aos temas mapeamento de processo e pensamento enxuto, apresentando as características das publicações junto à base de dados Web of Science, no período correspondente de 2010 a 2019.

\section{MAPEAMENTO DE PROCESSO}

Mapeamento de processo é a descrição de como os processos e as atividades relacionam-se, detalhando o fluxo de materiais, pessoas e informações, permitindo a compressão de todo o macroprocesso. Além do que, o fluxograma é uma das formas de representar graficamente as atividades, no qual é possível identificar problemas e pontos de melhoria (Slack, Chambers \& Johnston, 2006).

Além do mais, o mapeamento de processos é uma forma de organizar um processo ou setor em forma de diagrama, permitindo análises de seu funcionamento e possíveis alterações em busca de otimização. Para a realização de melhorias em um processo, se faz necessário a visualização das diversas etapas que o constituem, bem como suas interações e sequência cronológica, podendo ser representado visualmente através de fluxogramas (Barbrow \& Hartline, 2015).

Dito isso, o mapeamento de processos precisa ser desempenhado de forma que, ao se mapear ou desenhar os processos, todas as atividades que não agregam valor do ponto de vista do cliente devem ser eliminadas, além de corrigir as falhas do processo e desempenho que ocasionam retrabalhos e perda de qualidade (Rocha \& Albuquerque, 2007).

As transformações que acontecem em determinado processo, devem adicionar valor ao produto, e gerar resultados que sejam mais eficientes ao cliente. As melhorias geradas por essas transformações resultam na melhoria dos processos e consequentemente do produto final, aumentando desta forma sua qualidade (Rossi, Prim, Bem \& Dandolin, 2017).

\section{Pensamento enxuto}

A Produção Enxuta surgiu entre os anos de 1949 a 1950 com Eiji Toyoda e Taiichi Ohno. Ohno, ao realizar visitas às instalações da Ford em Detroit, nos Estados Unidos da América, identificou vários desperdícios no processo de produção em massa. À vista disso, Ohno desenvolveu meios para combater os desperdícios no processo produtivo da empresa Toyota. Para tanto, foram realizadas iniciativas, sendo elas: agrupar os trabalhadores em equipes; tornar as equipes polivalentes; proporcionar que as equipes recomendem melhorias nos processos produtivos; permitir autonomia para os trabalhadores tomarem decisões em caso de ocorrência de problemas e buscarem, em equipe, a solução (Womack, Jones \& Roos, 2004). Por meio dessas iniciativas, a Toyota diminuiu drasticamente seu índice de reparos, além de aumentar a qualidade dos produtos acabados, e a diminuir os custos de produção.

O pensamento enxuto é uma forma de especificar valor ao produto ou serviço, alinhando sequências das ações que o melhore. Além disso, as atividades devem ser executadas sem interrupções sempre que solicitada, de forma eficiente e atendendo os cinco princípios básicos: valor; fluxo de Valor; fluxo; produção puxada; e perfeição (Womack \& Jones, 1997). 

Brazilian Journal of Production Engineering, 6(9), Edição Especial "Iniciação Científica", 47-65.

No pensamento enxuto, entregar a qualidade esperada pelo cliente se torna o objetivo central da organização. Deve-se considerar a qualidade esperada pelo cliente globalmente no ambiente empresarial e não apenas na área do produto e produção. Utilizando a abordagem da qualidade como estratégia no gerenciamento de projeto do produto, garante-se maior sucesso no atendimento das necessidades do cliente, atingindo desta forma sucesso em diversas frentes (Stone, 2012).

\section{Método de Pesquisa}

O presente estudo foi desenvolvido por meio de pesquisa bibliométrica, a fim de ampliar o conhecimento e analisar a quantidade de pesquisas referente às publicações relacionadas aos temas: Mapeamento de processos (process mapping); e Pensamento enxuto (lean thinking). Além disso, foi realizada uma pesquisa descritiva com uma abordagem quantitativa dos dados encontrados.

A pesquisa descritiva tem a função de tratar de aspectos gerais e amplos de um contexto, desenvolvendo uma análise que identifique as diferentes formas dos fenômenos. Ainda, a abordagem do artigo se caracteriza como quantitativa, na qual utiliza-se instrumentos estatísticos, tanto na coleta quanto no tratamento dos dados (Gerhardt \& Silveira, 2009).

Os dados para elaboração da referida pesquisa foram coletados na base Web of Science do Institute for Scientific Information (ISI). Além do mais, por meio do mecanismo de pesquisas, foram utilizados como palavras-chave "process mapping" e "lean thinking", delimitando a busca para o período de 2010 - 2019, isto é, últimos dez anos. Salienta-se que as palavraschave foram colocadas nos campos de pesquisas entre aspas, buscando garantir que os artigos encontrados possuam em seus textos as palavras da forma em que foram pesquisadas. Isso foi feito, pois como elas se tratam de palavras compostas e nas outras tentativas de buscas, nas quais as palavras estavam sem aspas apareceram artigos relacionados apenas a vários tipos de mapeamentos, e pensamentos que não tinham nenhuma ligação com os temas.

Ademais, foram definidos como parâmetros arquivos que fossem artigos de acesso livre. Além disso, foram analisadas pesquisas que abordavam os temas separadamente e em conjunto. Foram encontrados 115 artigos relacionados ao process mapping, 80 a lean thinking e 3 utilizando ambos.

O desenvolvimento da pesquisa ocorreu através da análise gráfica fornecida pela Web of Science, sendo considerados sete critérios: autores; áreas de pesquisa; anos de publicação; organizações; países/regiões; agências financiadoras; e fontes de publicações. Além disso, foram analisadas as quantidades de citações anualmente e palavras-chave relacionadas aos temas, sendo este explorado graficamente pelo software VOSviewer, em que os dados foram exportados da Web of Science e utilizados como entrada pela o software. Ainda, os demais gráficos e tabelas foram elaborados utilizando o software Microsoft Excel, que utilizou a base de dados como entrada de informações.

Desse modo, após análise bibliométrica do referido estudo, foram levantadas as áreas de pesquisa, autores, universidades, agências financiadoras e países que mais publicam sobre os 
temas. Assim, foi possível ter uma melhor compreensão quanto a presença destes temas no meio cientifico.

\section{ANÁLISE E DISCUSSÃo DOS RESULTADOS}

Verificou-se 198 artigos ao todo, relacionados aos temas na base Web of Science, no período de tempo estipulado. Além do mais, apresentou-se as principais características das publicações relacionadas aos temas de acordo aos critérios expostos na metodologia.

\subsection{AUTORES}

Como mostrado nas Tabelas 1, 2 e 3 os autores que mais publicaram sobre process mapping e lean thinking, tiveram 2 e 3 artigos publicados respectivamente. Após realizar o levantamento dos dados, foi encontrada uma grande quantidade de autores, com algumas publicações sobre os temas, nos dez anos analisados.

Tabela 1. Autores e quantidade de artigos publicados relacionados ao process mapping.

\begin{tabular}{llc}
\hline A. & QUTORES & 2 \\
\hline 1. & BLVES GK & 2 \\
2. BHATTACHARYA A & 2 \\
3. BLAKELY B & 2 \\
4. BOI L & 2 \\
5. BRKIC L & 2 \\
6. CLARKE DJ & 2 \\
7. CLAY-WILLIAMS R & 2 \\
8. DINIS-CARVALHO J & 2 \\
9. DRUMMOND A & 2 \\
10. FORSTER A & 2 \\
11. GRENFELL K & 2 \\
12. KAPLAN RS & 2 \\
13. LANE P & 2 \\
14. PALMER R & 2 \\
15. PRESCOTT M & 2 \\
16. RODGERS H & 2 \\
17. SENTHURAN S & 2 \\
18. TYRRELL P & 2 \\
19. WEINER BJ & 1 \\
20. ABD ALRIDHA AM & 1 \\
21. ABMAN SH & 1 \\
22. ABOUZAHR C & 1 \\
23. AGARWAL N & 1 \\
24. AGHA R & 1 \\
25. AHMAD T &
\end{tabular}

Fonte: Autores (2020).

Tabela 2. Autores e quantidade de artigos publicados relacionados ao lean thinking.

\begin{tabular}{|c|c|}
\hline AUTORES & QUANTIDADE D \\
\hline 1. Radnor Z & 3 \\
\hline 2. Boak G & 2 \\
\hline 3. Caiado Rgg & 2 \\
\hline 4. Cesarelli M & 2 \\
\hline 5. Da Silva El & 2 \\
\hline 6. Dos Santos Jlg & 2 \\
\hline 7. Erdmann $\mathrm{Al}$ & 2 \\
\hline 8. Freitas Mdd & 2 \\
\hline 9. Granja Ad & 2 \\
\hline 10. Improta $G$ & 2 \\
\hline
\end{tabular}




\begin{tabular}{ll} 
11. Ivson P & 2 \\
12. Koskela L & 2 \\
13. Mazzocato P & 2 \\
14. Nascimento Dld & 2 \\
15. Romano M & 2 \\
16. Sermeus W & 2 \\
17. Sol Jca & 2 \\
18. Tezel A & 2 \\
19. Van Vliet Ej & 2 \\
20. Vlachos I & 2 \\
21. Abu-Khudair H & 1 \\
22. Abushaikha I & 1 \\
23. Agnetis A & 1 \\
24. Ahmad W & 1 \\
25. Aij Kh & 1 \\
\hline
\end{tabular}

Fonte: Autores (2020).

Tabela 3. Autores e quantidade de artigos publicados relacionados ao process mapping e lean thinking.

\begin{tabular}{l|c}
\hline \multicolumn{1}{c|}{ AUTORES } & QUANTIDADE DE ARTIGOS \\
\hline 26. Antony J & 1 \\
27. Balato G & 1 \\
28. Camarini G & 1 \\
29. Cesarelli M & 1 \\
30. Cuccaro P & 1 \\
31. Douglas A & 1 \\
32. Douglas Ja & 1 \\
33. Euphrosino Ca & 1 \\
34. Fontanini Psp & 1 \\
35. Improta G & 1 \\
36. Ortigara Yvb & 1 \\
37. Pimentel Ll & 1 \\
38. Ponsiglione Am & 1 \\
39. Raiola E & 1 \\
40. Romano M & 1 \\
41. Ruiz Pv & 1 \\
42. Russo Ma & 1 \\
43. Santillo Lc & 1 \\
\hline
\end{tabular}

Fonte: Autores (2020).

Os autores Mario Cesarelli (Cesarelli M.), Giovanni Improta (Improta G.) e Maria Romano (Romano M.), foram os únicos que além de estarem presentes em um dos dois temas individualmente, também estiveram nas publicações envolvendo as duas temáticas. Os dois primeiros autores são da University of Naples Federico II, localizada em Nápoles, Itália. O Romano M trabalha na unidade dermatológica do Osped S Camillo Roma, localizado em Roma, Itália.

\section{2. ÁREAS DE PESQUISA}

Como observado nas Tabelas 4, 5 e 6, sobre às áreas do conhecimento que abrangem a temática presentes no Web of Science com relação ao process mapping, demonstra-se que Engenharia (Engineering), Serviços de ciências da saúde (Health care sciences services), Medicina Geral interna (General internal medicine) e Economia de negócios (Business economics), são aquelas que obtiveram maior número de publicações. Em lean thinking, as áreas foram: Engenharia (Engineering); Serviços de ciências da saúde (Health care sciences

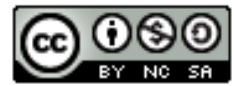



Brazilian Journal of Production Engineering, 6(9), Edição Especial "Iniciação Científica", 47-65.

services); Economia de negócios (Business economics); e Ciência de gerenciamento de pesquisa operacional (Operations research management science). Analisando publicações que envolviam ambos os temas em conjunto, foram encontradas as seguintes áreas de pesquisa: Economia de negócios (Business economics); Medicina interna geral (General internal medicine); Serviços de ciências da saúde (Health care sciences services); Ciência dos materiais (Materials science); e Informação médica (Medical informatics).

Tabela 4. Áreas de pesquisas: process mapping.

\begin{tabular}{l|c}
\hline \multicolumn{1}{c}{ ÁREAS } & QUANTIDADE DE ARTIGOS \\
\hline ENGINEERING & 17 \\
HEALTH CARE SCIENCES SERVICES & 15 \\
GENERAL INTERNAL MEDICINE & 14 \\
BUSINESS ECONOMICS & 13 \\
OUTRAS ÁREAS & 69 \\
\hline
\end{tabular}

Fonte: Autores (2020).

Tabela 5. Áreas de pesquisas: lean thinking.

\begin{tabular}{l|c}
\hline \multicolumn{1}{c}{ ÁREAS } & QUANTIDADE DE ARTIGOS \\
\hline ENGINEERING & 28 \\
HEALTH CARE SCIENCES SERVICES & 15 \\
BUSINESS ECONOMICS & 14 \\
OPERATIONS RESEARCH MANAGEMENT SCIENCE & 8 \\
OUTRAS ÁREAS & 39 \\
\hline
\end{tabular}

Fonte: Autores (2020).

Tabela 6. Áreas de pesquisas: process mapping e lean thinking.

\begin{tabular}{l|c}
\hline \multicolumn{1}{c}{ ÁREAS } & QUANTIDADE DE ARTIGOS \\
\hline BUSINESS ECONOMICS & 1 \\
GENERAL INTERNAL MEDICINE & 1 \\
HEALTH CARE SCIENCES SERVICES & 1 \\
MATERIALS SCIENCE & 1 \\
MEDICAL INFORMATICS & 1 \\
\hline
\end{tabular}

Fonte: Autores (2020).

As áreas que ocupam as primeiras posições no ranking de publicações apresentado sugerem uma evidente preocupação maior da Engenharia em estudar sobre os temas isoladamente, porém ao estudar os temas em conjunto há presença de cinco áreas de pesquisa, entretanto nenhuma delas é a Engenharia, diferente do que ocorreu nos outros dois casos, isso demonstra que outras áreas tomam a iniciativa em relação às pesquisas envolvendo ambos os temas em conjunto.

\subsection{ANOS DE PUBLICAÇÃOO}

No período compreendido entre 2010 e 2019, foram levantadas a quantidade de publicações a cada ano. As publicações referentes ao process mapping são observadas na Figura 1 e lean thinking na Figura 2. Publicações referentes aos artigos que envolviam ambos os temas em conjunto não tiveram boa expressão, tendo somente três artigos publicados, um em 2015 e outros dois em 2017 e 2019, respectivamente. Esta análise demonstra a necessidade de pesquisas envolvendo os temas em conjunto, já que nos últimos dez anos tiveram apenas três publicações.

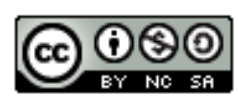


Analisando separadamente cada tema, conforme a Figura 1, o ano 2019 foi o mais expressivo em relação às publicações sobre o process mapping, além de um comportamento crescente entre os anos de 2014 a 2019, que resultaram em um aumento em pesquisas relacionadas ao tema. Como mostrado na Figura 2, o lean thinking apresentou um aumento nas publicações entre os anos de 2014 a 2018, porém no último ano, pesquisas relacionadas ao tema tiveram uma acentuada queda, sendo que em 2018 houve 21 publicações, já em 2019 apenas 7 artigos.

Figura 1. Publicações referentes ao process mapping.

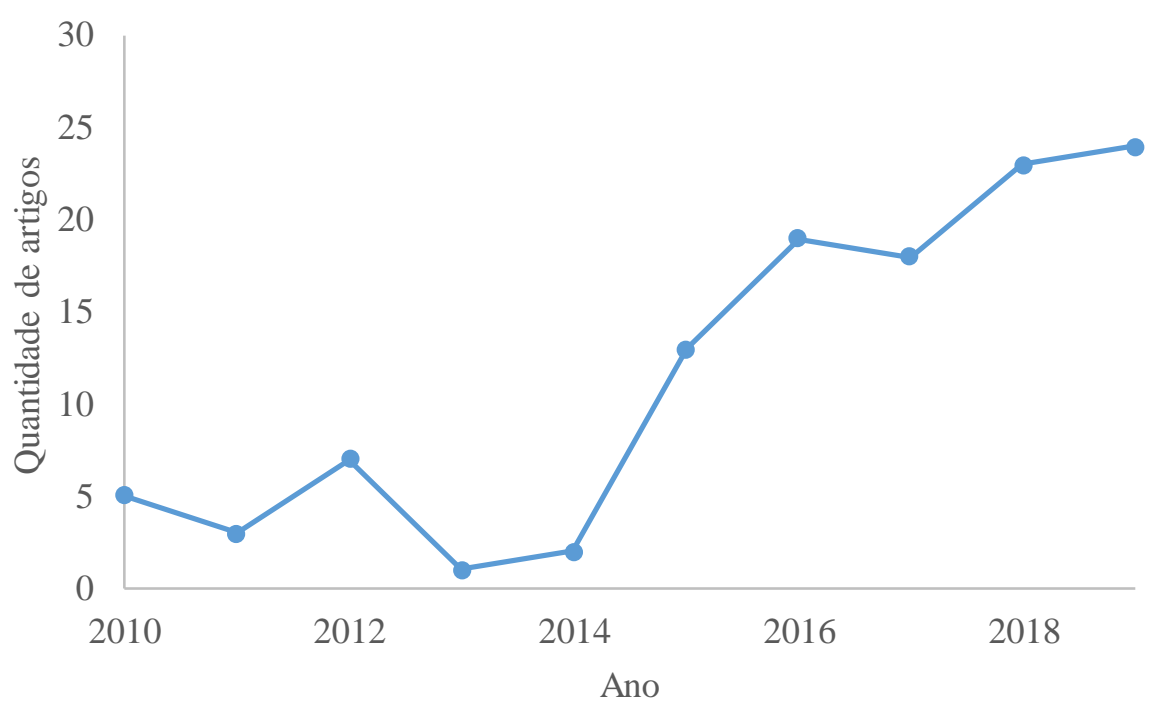

Fonte: Autores (2020).

Figura 2. Publicações referentes ao lean thinking.

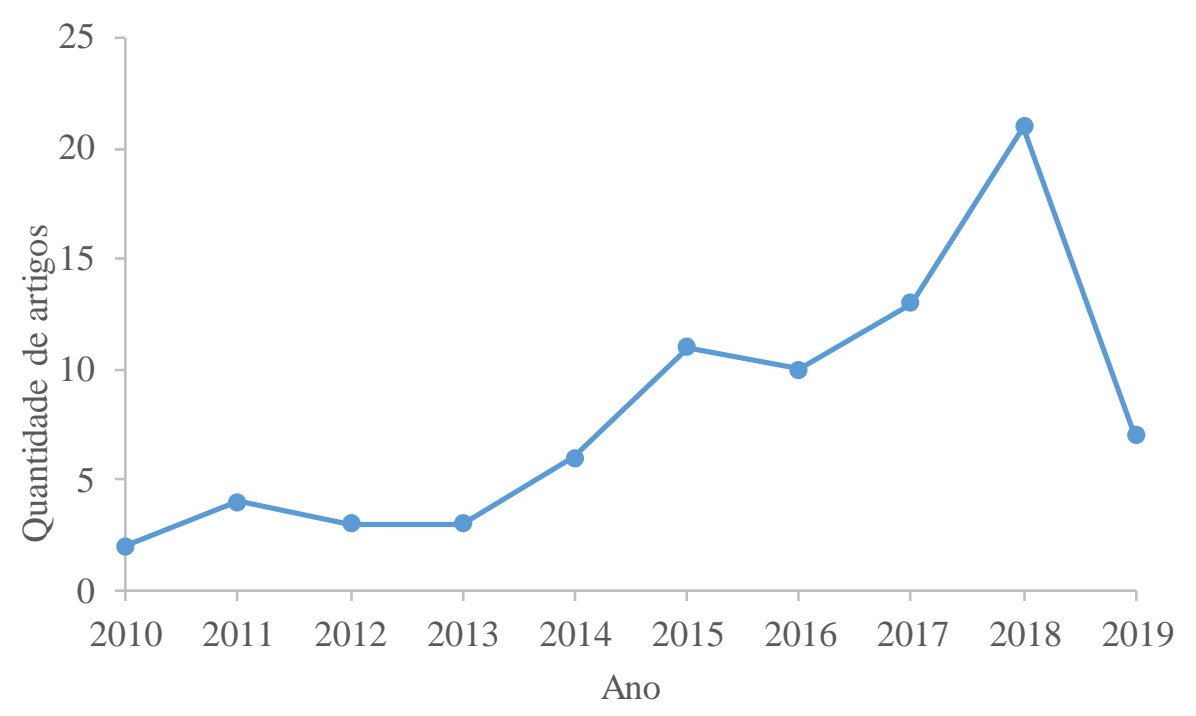

Fonte: Autores (2020).

\subsection{ORgANIZAÇÕES}

As instituições que mais publicaram artigos relacionados aos temas estão presentes nas Tabelas 7, 8 e 9. Conforme mostrado na Tabela 7, entre as instituições relacionadas ao process mapping, a University of London, localizada em Londres, Inglaterra, é a organização 
que mais publicou nos últimos dez anos. De acordo a Tabela 8, entre as instituições relacionadas ao lean thinking, a Universidade Estadual de Campinas, localizada em Campinas - SP, foi a que mais se destacou nos últimos dez anos. Com relação às pesquisas envolvendo ambos os temas em conjunto, não tiveram nenhuma organização em destaque, pois todas publicaram a mesma quantidade de artigos, conforme demonstrado na Tabela 9.

Tabela 7. Organizações: process mapping.

\begin{tabular}{l|c}
\hline ORGANIZAÇÕES & QUANTIDADE DE ARTIGOS \\
\hline UNIVERSITY OF LONDON & 8 \\
HARVARD UNIVERSITY & 4 \\
LONDON SCHOOL OF HYGIENE TROPICAL MEDICINE & 4 \\
UNIVERSIDADE FEDERAL DE SANTA CATARINA UFSC & 4 \\
UNIVERSITY COLLEGE LONDON & 4 \\
UNIVERSITY OF CALIFORNIA SYSTEM & 4 \\
UNIVERSITY OF COLORADO HEALTH SCIENCE CENTER & 4 \\
UNIVERSITY OF COLORADO SYSTEM & 4 \\
UNIVERSITY OF MANCHESTER & 4 \\
UNIVERSITY OF TORONTO & 4 \\
MACQUARIE UNIVERSITY & 3 \\
UNIVERSIDADE DE SAO PAULO & 3 \\
UNIVERSITY OF BIRMINGHAM & 3 \\
UNIVERSITY OF CALGARY & 3 \\
UNIVERSITY OF MELBOURNE & 3 \\
UNIVERSITY OF NOTTINGHAM & 3 \\
UNIVERSITY OF OXFORD & 3 \\
ASTON UNIVERSITY & 2 \\
BRADFORD TEACHING HOSP NHS TRUST & 2 \\
CHILDREN S HOSPITAL COLORADO & 2 \\
CINCINNATI CHILDREN S HOSPITAL MEDICAL CENTER & 2 \\
CRANFIELD UNIVERSITY & 2 \\
DARTMOUTH COLLEGE & 2 \\
KAROLINSKA INSTITUTET & 2 \\
MCMASTER UNIVERSITY & 2 \\
\hline
\end{tabular}

Fonte: Autores (2020).

Tabela 8. Organizações: lean thinking.

\begin{tabular}{lc}
\hline \multicolumn{1}{c}{ ORGANIZAÇÕES } & QUANTIDADE DE ARTIGOS \\
\hline UNIVERSIDADE ESTADUAL DE CAMPINAS & 5 \\
CARDIFF UNIVERSITY & 2 \\
KAROLINSKA INSTITUTET & 2 \\
LINKOPING UNIVERSITY & 2 \\
MAGNA GRAECIA UNIVERSITY OF CATANZARO & 2 \\
PONTIFICIA UNIVERSIDADE CATOLICA DO RIO DE JANEIRO & 2 \\
SWANSEA UNIVERSITY & 2 \\
TECNOLOGICO DE MONTERREY & 2 \\
UNIVERSIDADE DO ESTADO DE SANTA CATARINA & 2 \\
UNIVERSIDADE FEDERAL DE SANTA CATARINA UFSC & 2 \\
UNIVERSIDADE FEDERAL DO MARANHAO & 2 \\
UNIVERSIDADE FEDERAL FLUMINENSE & 2 \\
UNIVERSITY OF HUDDERSFIELD & 2 \\
UNIVERSITY OF LEICESTER & 2 \\
UNIVERSITY OF NAPLES FEDERICO II & 2 \\
UNIVERSITY OF NORTH CAROLINA & 2 \\
UNIVERSITY OF NORTH CAROLINA CHAPEL HILL & 2 \\
UNIVERSITY OF NOTTINGHAM & 2 \\
UNIVERSITY OF SALFORD & 2 \\
UNIVERSITY OF WARWICK & 2 \\
VIRGINIA POLYTECHNIC INSTITUTE STATE UNIVERSITY & 2 \\
YORK SAINT JOHN UNIVERSITY & 2 \\
5 BOROUGHS PARTNERSHIP NHS FDN TRUST & 1 \\
AALTO UNIVERSITY & 1 \\
ANADOLU UNIVERSITY & 1 \\
\hline
\end{tabular}

Fonte: Autores (2020).

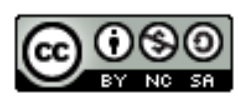



Brazilian Journal of Production Engineering, 6(9), Edição Especial "Iniciação Científica", 47-65.

Tabela 9. Organizações: process mapping e lean thinking.

\begin{tabular}{lc}
\hline \multicolumn{1}{c}{ ORGANIZAÇõES } & QUANTIDADE DE ARTIGOS \\
\hline HERIOT WATT UNIVERSITY & 1 \\
INSTITUTO FEDERAL DO SUL DE MINAS GERAIS & 1 \\
IRCCS FONDAZIONE SALVATORE MAUGERI & 1 \\
LIVERPOOL JOHN MOORES UNIVERSITY & 1 \\
MAGNA GRAECIA UNIVERSITY OF CATANZARO & 1 \\
MANAGEMENT UNIV AFRICA & 1 \\
PONTIFICIA UNIVERSIDADE CATOLICA DE & 1 \\
CAMPINAS & 1 \\
UNIV HOSP FEDERICO II & 1 \\
UNIVERSIDADE ESTADUAL DE CAMPINAS & 1 \\
UNIVERSITY OF LIVERPOOL & 1 \\
UNIVERSITY OF NAPLES FEDERICO II & \\
\hline
\end{tabular}

Fonte: Autores (2020).

A presença de uma universidade inglesa na liderança entre as instituições que mais publicaram sobre o process mapping, podem estar relacionadas ao fato que países onde predominam a língua inglesa foram os que mais publicaram sobre o tema, o que eleva o número de publicações das instituições locais. Ainda, a presença de uma universidade brasileira como a que mais publicou sobre lean thinking, pode estar relacionada a boa colocação do Brasil em publicações relacionadas ao tema ficando atrás apenas dos EUA e Inglaterra, que pode ser um impacto positivo do aumento de universitários o que resulta em um aumento de pesquisas acadêmicas.

\subsection{Países/Região}

Conforme observado na Tabela 10 os países que mais publicaram sobre process mapping, foram os Estados Unidos da América que lidera o ranking de publicações, seguidos pela Inglaterra e Brasil. Como mostrado na Tabela 11, os países que mais publicaram sobre lean thinking, são a Inglaterra que lidera o ranking de publicações, seguida pelo Brasil e Países Baixos. Ademais, apenas cinco países publicaram artigos que envolveram os dois temas em conjunto, sendo eles: Brasil; Inglaterra; Itália; Quênia; e Escócia.

Tabela 10. Organizações: process mapping.

\begin{tabular}{l|c}
\hline \multicolumn{1}{c|}{.PAÍSES/REGĨ̃o } & QUANTIDADE DE ARTIGOS \\
\hline ESTADOS UNIDOS DA AMÉRICA & 38 \\
INGLATERRA & 30 \\
BRASIL & 17 \\
CANADÁ & 7 \\
AUSTRÁLIA & 6 \\
\hline
\end{tabular}

Fonte: Autores (2020).

Tabela 11. Organizações: lean thinking.

\begin{tabular}{lc}
\hline \multicolumn{1}{c}{ PAÍSES/REGIÃo } & QUANTIDADE DE ARTIGOS \\
\hline INGLATERRA & 17 \\
BRASIL & 11 \\
PAÍSES BAIXOS & 7 \\
ESTADOS UNIDOS DA AMÉRICA & 7 \\
ITÁLIA & 5 \\
\hline
\end{tabular}

Fonte: Autores (2020).

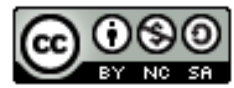



Brazilian Journal of Production Engineering, 6(9), Edição Especial "Iniciação Científica", 47-65.

Dessa forma, conforme o Web of Science nesses países se encontram a maioria das instituições que possuem pesquisas relacionadas aos temas. Destaca-se que o Brasil ocupa posições importantes no ranking das publicações relacionadas ao tema pesquisado. Ao todo foram 171 publicações, totalizando $86,36 \%$ do total dos artigos publicados, esse crescimento pode estar relacionado ao aumento de estudantes em universidades durante o período do presente estudo, o que pode resultar no crescimento de pesquisas acadêmicas.

\subsection{AGÊNCIAS FINANCIADORAS}

Na Tabela 12 são apresentadas todas as agências financiadoras de pesquisas relacionadas ao process mapping, note que a United States Department of Health Human Services (Departamento de Saúde e Serviços Humanos dos Estados Unidos) e o National Institutes of Health Nih USA, foram às agências que mais se destacaram. Com sede em Washington, D.C., nos Estados Unidos da América, a United States Department of Health Human Services investem em meios de proteger a saúde de todos os estadunidenses e prover serviços humanos essenciais, parte desses investimentos são direcionados a pesquisas (HHS, 2020). Com sede em Rockville, Maryland, EUA, o National Institutes of Health USA são um conglomerado de centros de pesquisa que formam a agência governamental de pesquisa biomédica do Departamento de Saúde e Serviços Humanos dos Estados Unidos (NIH, 2020).

Tabela 12. Agências financiadoras: process mapping.

\begin{tabular}{lcc}
\hline \multicolumn{1}{c}{ AGÊNCIAS FINANCIADORAS } & QUANTIDADE DE ARTIGOS \\
\hline UNITED STATES DEPARTMENT OF HEALTH HUMAN SERVICES & 10 \\
NATIONAL INSTITUTES OF HEALTH NIH USA & 9 \\
NATIONAL INSTITUTE FOR HEALTH RESEARCH NIHR & 4 \\
ENGINEERING PHYSICAL SCIENCES RESEARCH COUNCIL EPSRC & 3 \\
EUROPEAN UNION EU & 2 \\
GATES FOUNDATION & 2 \\
NIH NATIONAL CANCER INSTITUTE NCI & 2 \\
NIH NATIONAL CENTER FOR ADVANCING TRANSLATIONAL SCIENCES NCATS & 2 \\
NIH NATIONAL INSTITUTE OF DIABETES DIGESTIVE KIDNEY DISEASES NIDDK & 2 \\
OFFICE OF RURAL HEALTH ORH & 2 \\
TOWNSVILLE HOSPITAL AND HEALTH SERVICE RESEARCH TRUST FUND & 2 \\
AARHUS UNIVERSITY HOSPITAL & 1 \\
AFRICAN DOCTORAL DISSERTATION RESEARCH FELLOWSHIP ADDRF & 1 \\
AGENCY FOR HEALTHCARE RESEARCH QUALITY & 1 \\
ANDALUSIA GOVERNMENT & 1 \\
AUSTIN HEALTH & 1 \\
AUSTRALIAN GENOME RESEARCH FACILITY & 1 \\
BANNER HEALTH RISK MANAGEMENT & 1 \\
FUND PROGRAM & 1 \\
BRITISH COUNCIL UNDER UK INDIA EDUCATION AND RESEARCH INITIATIVE & 1 \\
UKIERI & 1 \\
CANCER CENTER SUPPORT GRANT NCI & 1 \\
CANCER RESEARCH UK & 1 \\
PENTERS FOR DISEASE CONTROL AND PREVENTION DIVISION OF LABORATORY & 1 \\
CENTERS FOR DISEASE CONTROL PREVENTION USA & 1 \\
CENTRAL REGION DENMARK & 1 \\
CHIEF SCIENTIST OFFICE & 1 \\
\hline
\end{tabular}

Fonte: Autores (2020).

Na Tabela 13 são apresentadas todas as agências financiadoras de pesquisas relacionadas ao lean thinking, note que a Coordenação de Aperfeiçoamento de Pessoal de Nível Superior (CAPES) lidera este ranking, fundada em julho de 1951 a CAPES possui suas atividades agrupadas nas seguintes linhas e ações: avaliação da pós-graduação stricto sensu; acesso e 

Brazilian Journal of Production Engineering, 6(9), Edição Especial "Iniciação Científica", 47-65.

divulgação da produção científica; investimentos na formação de recursos humanos de alto nível, no país e exterior; promoção da cooperação científica internacional; e indução e fomento da formação inicial e continuada de professores para a educação básica nos formatos presencial e a distância (CAPES, 2019).

Tabela 13. Agências financiadoras: lean thinking.

\begin{tabular}{|c|c|}
\hline AGÊNCIAS FINANCIADORAS & $\begin{array}{l}\text { QUANTIDADE DE } \\
\text { ARTIGOS }\end{array}$ \\
\hline $\begin{array}{l}\text { COORDENAÇÃO DE APERFEIÇOAMENTO DE PESSOAL DE } \\
\text { NÍVEL SUPERIOR }\end{array}$ & 2 \\
\hline AFA FORSAKRING & 1 \\
\hline $\begin{array}{l}\text { BRAZILIAN FEDERAL AGENCY FOR SUPPORT AND } \\
\text { ASSESSMENT OF POST GRADUATE EDUCATION CAPES }\end{array}$ & 1 \\
\hline DEPARTMENT OF INDUSTRIAL ENGINEERING & 1 \\
\hline $\begin{array}{l}\text { DEPARTMENT OF SUSTAINABLE BIOMATERIALS AT VIRGINIA } \\
\text { TECH }\end{array}$ & 1 \\
\hline DIRECTION OF POLICLINICO SANTA MARIA ALLE SCOTTE & 1 \\
\hline ECONOMIC SOCIAL RESEARCH COUNCIL ESRC & 1 \\
\hline $\begin{array}{l}\text { ENGINEERING PHYSICAL SCIENCES RESEARCH COUNCIL } \\
\text { EPSRC }\end{array}$ & 1 \\
\hline $\begin{array}{l}\text { EUROPEAN UNION OPERATIONAL PROGRAMME II COHESION } \\
\text { POLICY INVESTING IN HUMAN CAPITAL TO CREATE MORE } \\
\text { OPPORTUNITIES AND PROMOTE THE WELLBEING OF SOCIETY } \\
\text { EUROPEAN SOCIAL FUND }\end{array}$ & 1 \\
\hline FINNISH INSTITUTE OF OCCUPATIONAL HEALTH & 1 \\
\hline FINNISH WORK ENVIRONMENT FUND & 1 \\
\hline FOPESQ & 1 \\
\hline $\begin{array}{l}\text { FUNDACAO DE AMPARO A PESQUISA DO ESTADO DE SAO } \\
\text { PAULO FAPESP }\end{array}$ & 1 \\
\hline GATES FOUNDATION & 1 \\
\hline GIESKES STRIJBIS FONDS & 1 \\
\hline HIGHWAYS ENGLAND & 1 \\
\hline LIDZ A DUTCH NETWORK FOR LEAN HEALTHCARE & 1 \\
\hline METAMETRICS INC & 1 \\
\hline $\begin{array}{l}\text { NATIONAL COUNCIL FOR SCIENTIFIC AND TECHNOLOGICAL } \\
\text { DEVELOPMENT CNPQ }\end{array}$ & 1 \\
\hline NETHERLANDS GOVERNMENT & 1 \\
\hline NOVUS GRANT & 1 \\
\hline $\begin{array}{l}\text { OFFICE OF SCIENTIFIC AFFAIRS AND RESEARCH AT KING } \\
\text { HUSSEIN CANCER CENTER }\end{array}$ & 1 \\
\hline $\begin{array}{l}\text { PORTUGUESE FOUNDATION FOR SCIENCE AND TECHNOLOGY } \\
\text { FCT }\end{array}$ & 1 \\
\hline $\begin{array}{l}\text { PORTUGUESE FUNDS THROUGH THE CIDMA CENTER FOR } \\
\text { RESEARCH AND DEVELOPMENT IN MATHEMATICS AND } \\
\text { APPLICATIONS }\end{array}$ & 1 \\
\hline $\begin{array}{l}\text { REACH HIGH SCHOLARS PROGRAMME POST DOCTORAL } \\
\text { GRANTS }\end{array}$ & 1 \\
\hline
\end{tabular}

Fonte: Autores (2020).

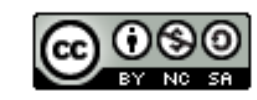



Brazilian Journal of Production Engineering, 6(9), Edição Especial "Iniciação Científica", 47-65.

Note que artigos relacionando aos temas em conjunto não foram representados, pois entre 2010 a 2019 não foram registradas nenhuma agência de financiamento relacionado ao mesmo. Além disso, as agências que mais se destacaram são norte-americanas, o que justifica a liderança dos Estados Unidos das Américas no ranking dos países que mais publicam sobre process mapping, conforme Tabela 10, e ter sido um dos destaques em lean thinking, conforme Tabela 11.

Ainda, tenha-se que 86,36\% dos artigos publicados foram no idioma inglês, isso se deve a dois fatores, primeiro é considerada a língua universal e segundo é que a maioria das agências de financiamentos estão localizadas em países em que possuem o idioma inglês como vernáculo. Além disso, os bancos de dados da Web of Science apresenta a maior parte de seus periódicos em língua inglesa.

\subsection{FONTES DE PUBLICAÇÕES}

De acordo a Tabela 14, a maioria dos estudos referentes aos temas foram publicados nos periódicos BMJ Open, BMC Health Services Research, International Journal for Quality in Health Care e Production Planning Control. Ademais, a maioria dos periódicos que se destacaram são relacionados a área da saúde, o que denota a relevância deste tema e a sua ampla aplicação.

Tabela 14. Principais fontes de publicações.

\begin{tabular}{l|c|c}
\hline \multicolumn{1}{c|}{ FONTES DE PUBLICAÇÕES } & QUANTIDADE DE ARTIGOS & TEMA RELACIONADO \\
\hline BMJ OPEN & 8 & PROCESS MAPPING \\
BMC HEALTH SERVICES RESEARCH & 4 & PROCESS MAPPING \\
BMC INFECTIOUS DISEASES & 2 & PROCESS MAPPING \\
BMC HEALTH SERVICES RESEARCH & 4 & LEAN THINKING \\
INTERNATIONAL JOURNAL FOR QUALITY IN & 4 & LEAN THINKING \\
HEALTH CARE & & LEAN THINKING \\
PRODUCTION PLANNING CONTROL & 4 & PROCESS MAPPING E \\
INTERNATIONAL JOURNAL OF QUALITY & 1 & LEAN THINKING \\
RELIABILITY MANAGEMENT & & PROCESS MAPPING E \\
JOURNAL OF EVALUATION IN CLINICAL & 1 & LEAN THINKING \\
PRACTICE & & PROCESS MAPPING E \\
MATERIA RIO DE JANEIRO & 1 & LEAN THINKING \\
\hline
\end{tabular}

Fonte: Autores (2020).

Ainda, esse destaque de periódicos relacionados a área da saúde podem ter sido motivados pelo avanço da medicina nos últimos anos, e ferramentas que permitem compreender de forma ampla todo processo e corrigir falhas e desperdícios são importantes para auxiliar nesses avanços.

\section{Citações}

Tendo por base a pesquisa sobre process mapping e lean thinking realizada na base Web of Science, as Tabelas 15 e 16 mostram as dez publicações mais citadas de cada tema. Essas publicações foram comparadas as Tabelas 1 e 2, que mostram os autores que mais 
Citação (APA): Amorim, M. de J., \& Xavier, T. P. (2021). Mapeamento de processo e pensamento enxuto: um estudo bibliométrico. Brazilian Journal of Production Engineering, 6(9), Edição Especial "Iniciação Científica", 47-65.

publicaram, para que fosse possível verificar se esses mesmos autores seriam os que mais tiveram os seus artigos utilizados.

Tabela 15. Citações: process mapping.

\begin{tabular}{|c|c|}
\hline PUBliCaÇões & $\begin{array}{c}\text { QUANTIDADE DE } \\
\text { CITAÇÕES }\end{array}$ \\
\hline $\begin{array}{l}\text { Overview Of Materials Qualification Needs For Metal Additive Manufacturing } \\
\text { PoR: Seifi, Mohsen; Salem, Ayman; Beuth, Jack; ET AL. } \\
\text { Jom VolumE: } 68 \text { EDIÇãO: } 3 \text { PÁGINAS: 747-764 PUBLICADO: MAR } 2016\end{array}$ & 128 \\
\hline $\begin{array}{l}\text { Steps Towards Sustainable Manufacturing Through Modelling Material, Energy And } \\
\text { Waste Flows } \\
\text { PoR: Smith, Leigh; Ball, Peter } \\
\text { International Journal Of Production } \\
\text { Economics VOLUME: } 140 \text { EDIÇÃO: } 1 \text { PÁGINAS: 227-238 PUBLICADO: NOV } 2012\end{array}$ & 116 \\
\hline 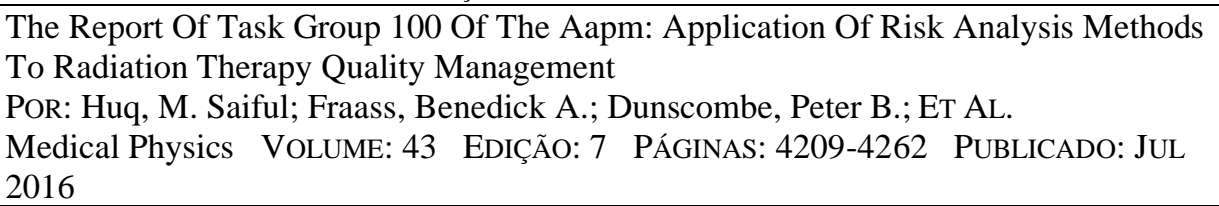 & 88 \\
\hline $\begin{array}{l}\text { Strategic Supplier Performance Evaluation: A Case-Based Action Research Of A Uk } \\
\text { Manufacturing Organisation } \\
\text { POR: Dey, Prasanta Kumar; Bhattacharya, Arijit; Ho, William } \\
\text { International Journal Of Production Economics VOLUME: } 166 \text { EDIÇÃO } \\
\text { ESPECIAL: SI PÁGINAS: 192-214 PUBLICADO: AUG 2015 }\end{array}$ & 50 \\
\hline $\begin{array}{l}\text { Time-Driven Activity-Based Costing: A Driver For Provider Engagement In Costing } \\
\text { Activities And Redesign Initiatives } \\
\text { PoR: Mclaughlin, Nancy; Burke, Michael A.; Setlur, Nisheeta P.; ET AL. } \\
\text { Neurosurgical Focus VOLUME: } 37 \text { EDIÇÃO: } 5 \text { NÚMERO DO } \\
\text { ARTIGO: E3 PUBLICADO: NOV } 2014\end{array}$ & 38 \\
\hline $\begin{array}{l}\text { Developing Crew Allocation System For The Precast Industry Using Genetic } \\
\text { Algorithms } \\
\text { POR: Al-Bazi, Ammar; Dawood, Nashwan } \\
\text { Computer-Aided Civil And Infrastructure } \\
\text { Engineering VOLUME: } 25 \text { EDIC̃ÃO: } 8 \text { PÁGINAS: 581-595 PUBLICADO: NOV } 2010\end{array}$ & 35 \\
\hline $\begin{array}{l}\text { Searching For The Missing Pieces Between The Hospital And Primary Care: Mapping } \\
\text { The Patient Process During Care Transitions } \\
\text { POR: Johnson, Julie K.; Farnan, Jeanne M.; Barach, Paul; ET AL. } \\
\text { AUTOR(ES) GRUPO: HANDOVER Res COLLABORATIVE } \\
\text { Bmj Quality \& Safety VOLUME: } 21 \text { SUPLEMENTO: } 1 \text { PÁGINAS: 97- } \\
105 \text { PUBLICADO: DEC } 2012\end{array}$ & 31 \\
\hline $\begin{array}{l}\text { Waste Identification And Elimination In Heis: The Role Of Lean Thinking } \\
\text { POR: Douglas, Jacqueline; Antony, Jiju; Douglas, Alexander } \\
\text { INTERNATIONAL JOURNAL OF QUALITY \& RELIABILITY } \\
\text { MANAGEMENT VOLUME: } 32 \text { EDIÇÃO: } 9 \text { EDIÇÃo ESPECIAL: SI PÁGINAS: 970- } \\
+\quad \text { PUBLICADO: } 2015\end{array}$ & 30 \\
\hline $\begin{array}{l}\text { Does The Process Map Influence The Outcome Of Quality Improvement Work? A } \\
\text { Comparison Of A Sequential Flow Diagram And A Hierarchical Task Analysis } \\
\text { Diagram } \\
\text { POR: Colligan, Lacey; Anderson, Janet E.; Potts, Henry W. W.; ET AL. } \\
\text { Bmc Health Services Research VOLUME: } 10 \text { NÚMERO DO } \\
\text { ARTIGO: } 7 \text { PUBLICADO: JAN } 72010\end{array}$ & 29 \\
\hline $\begin{array}{l}\text { INTEGRATING CANCER SURVIVORS' EXPERIENCES INTO UK CANCER REGISTRIES: } \\
\text { DESIGN AND DEVELOPMENT OF THE EPOCS SYSTEM (ELECTRONIC PATIENT-REPORTED } \\
\text { OUTCOMES FROM CANCER SURVIVORS) } \\
\text { POR: ASHLEY, L.; JONES, H.; THOMAS, J.; ET AL. } \\
\text { BRITISH JOURNAL OF CANCER VOLUME: } 105 \text { SUPLEMENTO: } 1 \text { PÁGINAS: S74- } \\
\text { S81 PUBLICADO: NOV } 32011\end{array}$ & 26 \\
\hline
\end{tabular}

Fonte: Autores (2020).

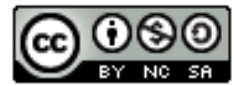

Esta obra está licenciada com uma Licença Creative Commons Atribuição-Não Comercial-Compartilha Igual 4.0 Internacional. Brazilian Journal of Production Engineering, São Mateus, Editora UFES/CEUNES/DETEC. 
Citação (APA): Amorim, M. de J., \& Xavier, T. P. (2021). Mapeamento de processo e pensamento enxuto: um estudo bibliométrico. Brazilian Journal of Production Engineering, 6(9), Edição Especial "Iniciação Científica", 47-65.

Tabela 16. Citações: Lean Thinking

Publicações QUANTIDADE DE CITAÇõES

LEAN IN HEALTHCARE: THE UNFILLED PROMISE?

POR: RADNOR, ZOE J.; HOLWEG, MATTHIAS; WARING, JUSTIN

SOCIAL SCIENCE \& MEDICINE VOLUME: 74 EDIÇÃO: 3 PÁGINAS: $364-$

371 PUBLICADO: FEB 2012

HOW DOES LEAN WORK IN EMERGENCY CARE? A CASE STUDY OF A LEAN-INSPIRED

INTERVENTION AT THE ASTRID LINDGREN CHILDREN'S HOSPITAL, STOCKHOLM,

SWEDEN

PoR: MaZZOCATO, PAMELA; Holden, Richard J.; BROMMEls, MATS; ET AL.

BMC HEALTH SERVICES RESEARCH VOLUME: 12 NÚMERO DO

ARTIGO: 28 PUBLICADO: FEB 12012

THE ROLE OF ACTOR ASSOCIATIONS IN UNDERSTANDING THE IMPLEMENTATION OF

LEAN THINKING IN HEALTHCARE

POR: PAPADOPOUlOS, THANOS; RADNOR, ZOE; MERALI, YASMIN

INTERNATIONAL JOURNAL OF OPERATIONS \& PRODUCTION

MANAGEMENT VOLUME: 31 EDIÇÃO: 1-2 PÁGINAS: 167-

191 PUBLICADO: 2011

APPLYING LEAN THINKING IN THE FOOD SUPPLY CHAINS: A CASE STUDY

POR: VLACHOS, ILIAS

PRODUCTION PLANNING \&

CONTROL Volume: 26 EdiçÃo: 16 PÁGINAs: 1351-1367 Publicado: DEC

102015

APPLYING VALUE STREAM MAPPING TO ELIMINATE WASTE: A CASE STUDY OF AN

ORIGINAL EQUIPMENT MANUFACTURER FOR THE AUTOMOTIVE INDUSTRY

Por: Lacerda, Antonio Pedro; Xambre, Ana Raquel; Alvelos, Helena

MARIA

INTERNATIONAL JOURNAL OF PRODUCTION

RESEARCH VOLUME: 54 EDIÇÃO: 6 PÁGINAS: 1708-

1720 PUBLICADO: MAR 182016

WASTE IDENTIFICATION AND ELIMINATION IN HEIS: THE ROLE OF LEAN THINKING

PoR: Douglas, JACQueline; ANTONY, JiJu; DOUGLAS, AlEXANDER

INTERNATIONAL JOURNAL OF QUALITY \& RELIABILITY

MANAGEMENT VOLUME: 32 EDIÇÃO: 9 EDIÇÃO

ESPECIAL: SI PÁGINAS: 970-+ PUBLICADO: 2015

LEAN THINKING IN THE EUROPEAN HOTEL INDUSTRY

POR: VLACHOS, ILIAS; BOGDANOVIC, ALEKSANDRA

TOURISM MANAGEMENT VOLUME: 36 PÁGINAS: 354-

363 PUBLICADO: JUN 2013

HOSPITAL CAPACITY PLANNING: FROM MEASURING STOCKS TO MODELLING FLOWS

Por: RECHEL, BERND; WRIGHT, STEPHEN; BARLOW, JAMES; ET AL.

BULLETIN OF THE WORLD HEALTH

ORGANIZATION VOLUME: 88 EDIÇÃO: 8 PÁGINAS: 632-

636 PUBLICADO: AUG 2010

EXPERIENCES OF LEADERS IN THE IMPLEMENTATION OF LEAN IN A TEACHING

HOSPITAL-BARRIERS AND FACILITATORS IN CLINICAL PRACTICES: A QUALITATIVE

STUDY

Por: Aij, KJeld Harald; Simons, Frederique Elisabeth; WidDERShOVEn,

GUY A. M.; ET AL.

BMJ OPEN VOLUME: 3 EDIÇÃO: 10 NÚMERO DO

ARTIGO: E003605 PUBLICADO: 2013

PROCESS IMPROVEMENT IN HEALTHCARE: OVERALl RESOURCE EFFICIENCY

POR: DE MAST, JEROEN; KEMPER, BENJAMIN; DOES, RONALD J. M. M.; ET AL.

QUALITY AND RELIABILITY ENGINEERING

INTERNATIONAL VOLUME: 27 EDIÇ̃̃o: 8 PÁGINAS: 1095-

1106 PUBLICADO: DEC 2011

Fonte: Autores (2020).

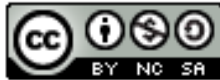

Esta obra está licenciada com uma Licença Creative Commons Atribuição-Não Comercial-Compartilha Igual 4.0 Internacional. Brazilian Journal of Production Engineering, São Mateus, Editora UFES/CEUNES/DETEC. 

Brazilian Journal of Production Engineering, 6(9), Edição Especial "Iniciação Científica", 47-65.

Conforme observado nas tabelas, foi possível constatar que apenas dois dos autores, sendo: Bhattacharya A. em process mapping; e Radnor Z. em lean thinking que mais publicaram e possuem seus trabalhos entre os 10 (dez) mais citados durante o período de tempo avaliado.

Portanto, as publicações com maior número de citações, que possivelmente são referências na área pesquisada, não pertencem aos autores que mais publicam sobre o mesmo tema que foram citados no tópico 5.1, isto é, autores com alto número de publicações não significa necessariamente que seja detentor dos artigos de maior impacto e relevância para a determinada área do conhecimento.

\section{Palavras-chave Relacionadas aOS temas}

Nas Figuras 3 e 4, são observadas as principais palavras-chave relacionadas aos temas. Salienta-se que foram adotados como parâmetros, palavras-chave que possuíam o número mínimo de ocorrência igual a três para process mapping e lean thinking, isto é, palavras que estiveram presentes em ao menos três artigos. Ainda, para gerar tais imagens foi utilizado o software VOSviewer

Figura 3. Rede de co-ocorrência de palavras-chave relacionada ao process mapping.

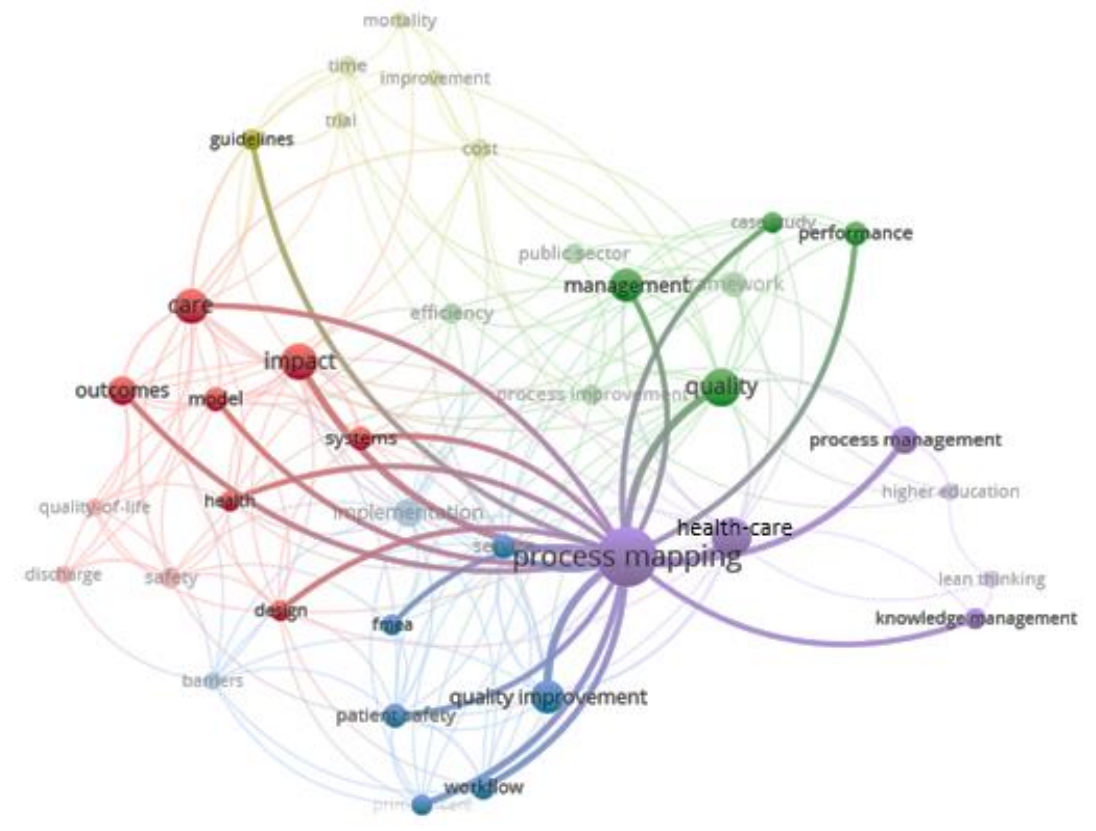

Fonte: Autores (2020).

Conforme a Figura 3, as palavras-chave que mais se relacionaram ao process mapping, foram: quality (Qualidade); health-care (Cuidados da saúde); care (Cuidado). Note, health-care está diretamente relacionada à área da saúde, ter uma das palavras-chave relacionada a este setor se justifica pelo fato da maioria dos periódicos que se destacaram serem relacionados a esta área, conforme abordado na categoria "5.7 Fontes de publicações" o que denota a relevância deste tema e a sua ampla aplicação.

Além disso, todas as palavras-chave fazem parte de áreas pesquisada pela United States Department Of Health Human Services e a National Institutes Of Health (NIH), que foram as principais agências financiadoras de pesquisas relacionadas ao process mapping, conforme tópico 5.6. Vale salientar que ambas as agências são do setor da saúde.

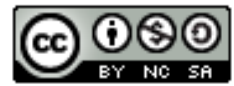



Brazilian Journal of Production Engineering, 6(9), Edição Especial "Iniciação Científica", 47-65.

Figura 4. Rede de co-ocorrência de palavras-chave relacionada ao lean thinking.

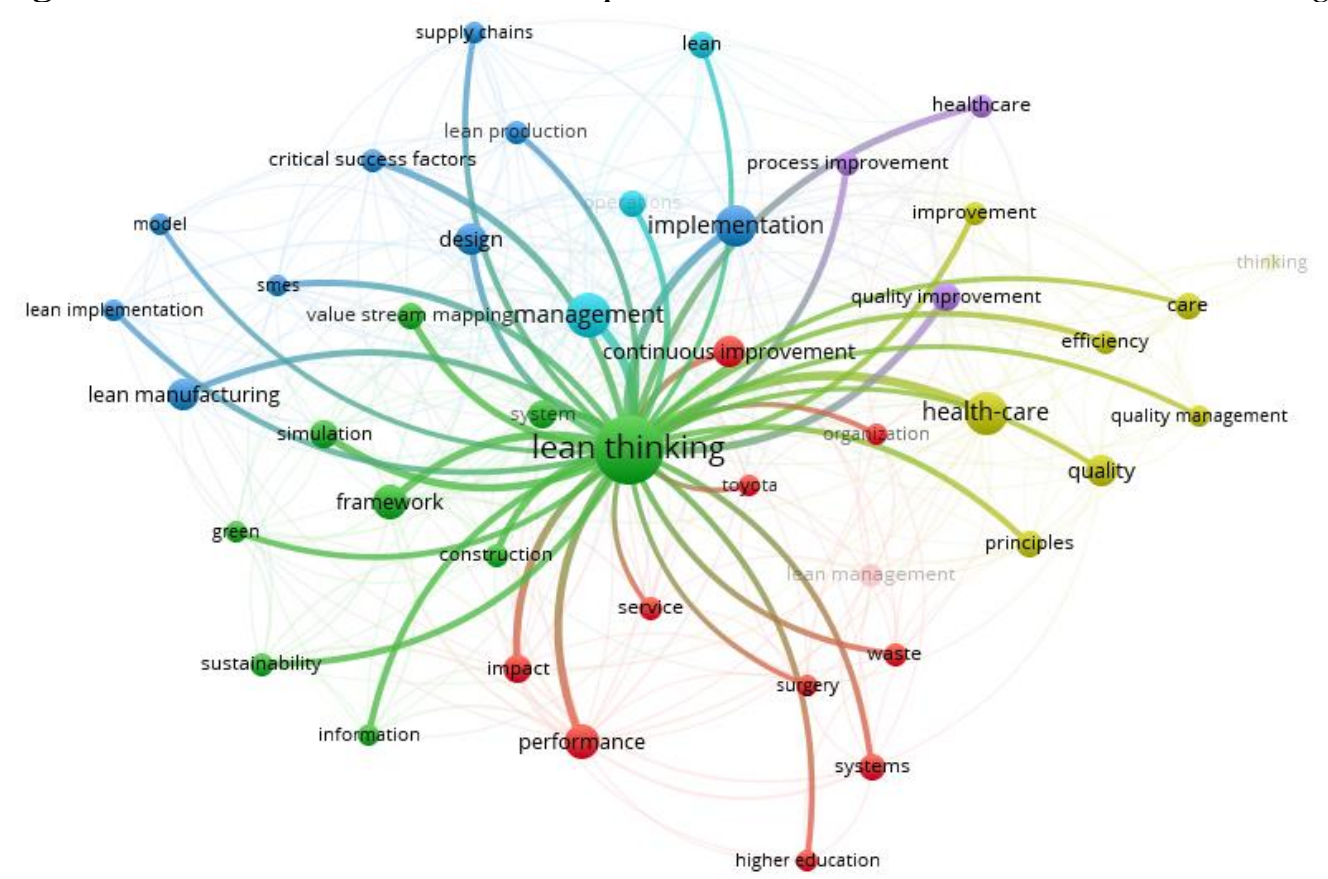

Fonte: Autores (2020).

Para o lean thinking de acordo com a Figura 4, as palavras-chave que mais se relacionaram ao tema, foram: management (Gestão); implementation (Implementação); health-care (Cuidados da saúde). Além disso, todas elas fazem parte de áreas pesquisada pela CAPES, que foi a principal agência financiada de pesquisas relacionadas ao lean thinking, conforme tópico 5.6. Salienta-se, a presença de algumas agências financiadoras relacionadas à saúde, o que justifica a presença de health-care entre as palavras-chave mais utilizadas no tema.

Considerando que foram encontrados apenas três artigos que relacionaram os dois temas em conjunto, e que estes não tiveram nenhuma agência financiadora, conforme o subtópico 5.6, as análises das palavras chaves não foram realizadas, pois o parâmetro para tal foi de que tivessem ao menos três ocorrências.

\section{CONSIDERAÇÕES FINAIS}

A pesquisa bibliométrica sobre process mapping e lean thinking na base de dados Web of Science evidenciou 198 publicações relacionadas a estes temas entre 2010-2019, destacandose as áreas temáticas engenharia (engineering), serviços de ciências da saúde (health care sciences services), medicina geral interna (general internal medicine), economia de negócios (business economics) e ciência de gerenciamento de pesquisa operacional (operations research management science).

Ademais, destaca-se a presença do Brasil entre os países que mais publicaram sobre os temas, ficando em terceiro lugar sobre process mapping e em segundo lugar sobre lean thinking. Os países que predominam a língua inglesa são os que mais se destacaram, sendo os Estados Unidos da América líderes no ranking dos países que mais publicaram sobre process mapping e a Inglaterra sobre lean thinking. Dessa forma, o idioma inglês é o predominante nas publicações, por dois principais motivos, primeiro é considerada a língua universal e segundo 
Citação (APA): Amorim, M. de J., \& Xavier, T. P. (2021). Mapeamento de processo e pensamento enxuto: um estudo bibliométrico. Brazilian Journal of Production Engineering, 6(9), Edição Especial "Iniciação Científica", 47-65.

os países que mais publicaram sobre os temas, possuem o idioma inglês como vernáculo. Ao todo foram 171 publicações, totalizando $86,36 \%$ do total de artigos publicados.

Além disso, são apresentadas todas as agências financiadoras de pesquisas relacionadas aos temas, sendo de maior destaque as norte-americanas, o que justifica a liderança dos Estados Unidos das Américas no ranking dos países que mais publicam sobre process mapping e lean thinking., porém assim como em outros tópicos, artigos relacionados aos temas em conjunto não tiveram muito destaque, pois entre 2010 a 2019 não foram registradas nenhuma agência de financiamento relacionadas ao mesmo.

Verificou-se também que os autores com os maiores números de publicações não são, necessariamente, os mais citados no período analisado, ou seja, as publicações que são possivelmente referências na temática pesquisada, não pertencem aos autores que mais publicam sobre os mesmos temas. Constatou-se que as publicações relacionadas ao process mapping tiveram um aumento entre 2014 a 2019, já o lean thinking teve esse aumento entre 2013 a 2018, porém no ano de 2019 teve uma brusca redução nos artigos publicados. A maioria dos periódicos que se destacaram ao número de publicações são relacionados à área da saúde, o que denota a relevância desse tema e a sua ampla aplicação.

Além da análise individual sobre os temas, foram realizados o levantamento de estudos envolvendo ambos os assuntos em conjunto, porém foram encontradas apenas três publicações nos últimos dez anos, o que limitou a análise. Diante ao exposto, entende-se que há um grande campo de trabalho para o desenvolvimento de pesquisas através de estudos envolvendo os temas abordados.

\section{REFERÊNCIAS}

Aguiar, W. S., Damasceno, M., \& Melo, F. (2016). Avaliação de softwares livres de bpmn para mapeamento de processos. In XII Congresso Nacional de Excelência Em Gestão \& III INOVARSE-Responsabilidade Social Aplicada.

Aherne, J., \& Whelton, J. (2010). Applying lean in healthcare: a collection of international case studies. New York: CRC Press.

Araújo, C. A. (2006). Bibliometria: evolução histórica e questões atuais. Em Questão, 12(1), $11-32$.

Barbrow, S., \& Hartline, M. (2015). Process mapping as organizational assessment in academic libraries. Performance Measurement and Metrics, 16.

CAPES - Coordenação de Aperfeiçoamento de Pessoal de nível Superior. (2019). História e missão. Recuperado em 23 de janeiro, 2020, de: https://www.capes.gov.br/historia-e-missao.

Gerhardt, T. E., \& Silveira, D. T. (2009). Métodos de Pesquisa. Plageder.

Gonçalves, A. C., Castro, P. R., Cruvinel, I. B., Jesus, R. S., Siqueira, D. C. B., \& Sousa, G. F. P. (2021). O Papel do Mapeamento de Processos - um estudo sobre a realização de exames periódicos da saúde em um órgão público. Brazilian Journal of Development, 7(3), 2127221296.

HHS - United States Department of Health Human Services. (2020). About HHS. Recuperado em 23 de janeiro, 2020, de: https://www.hhs.gov/about/index.html.

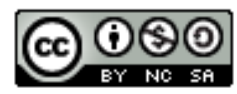


Hörbe, T. D. A. N., de Moura, G. L., Silva, A. H., Vargas, K. S., \& Machado, E. C. (2015). Gestão por Processos: Uma Proposta Aplicável a uma Pequena Empresa do Ramo de Alimentação. Sistemas \& Gestão, 10(2), 226-237.

Karim, A.; Uz-Zaman, K. A. (2013). A methodology for effective implementation of lean strategies and its performance evaluation in manufacturing organizations. Business Process Management Journal, 19(1), 169 - 196.

NIH - National Institutes of Health. (2020). A Short History of the National Institutes of Health. Recuperado em 23 de janeiro, 2020, de: https://history.nih.gov/exhibits/history/index.html.

Rocha, P., \& Albuquerque, A. (2007). Sincronismo organizacional. São Paulo: Saraiva.

Rossi, T., Prim, M. A., de Bem, R. M., \& Dandolin, G. A. (2017). Mapeamento de processos na BU/UFSC: aplicação do framework GC@BU. Revista Ibero-Americana de Ciência da Informação, 10(1), 204-217.

Slack, N., Chambers, S., \& Johnston. R. (2006). Administração da Produção. São Paulo: Atlas, 2006.

Stone, K. B. (2012). Four decades of lean: a systematic literature review. International Journal of Lean Six Sigma, 3(2), 112-132.

Vanti, N. (2002). Da bibliometria à webometria: uma exploração conceitual dos mecanismos utilizados para medir o registro da informação e a difusão do conhecimento. Ciência da Informação, 31(2), 152-162.

Womack, J. P., Jones, D. T., \& Roos, D. (2004). A máquina que mudou o mundo. Rio de Janeiro: Campus.

Womack, J. P., \& Jones, D. T. (1997). Lean thinking — banish waste and create wealth in your corporation. Journal of the Operational Research Society, 48(11), 1148-1148. 